\title{
ARTICLES
}

\section{Stability of viscoelastic shear flows subjected to parallel flow superposition}

\author{
V. V. Ramanan and M. D. Graham ${ }^{\mathrm{a})}$ \\ Department of Chemical Engineering and Rheology Research Center, University of Wisconsin-Madison, \\ Madison, Wisconsin 53706-1691
}

(Received 12 January 1999; accepted 2 August 2000)

\begin{abstract}
Steady viscoelastic shear flows in curved geometries are susceptible to instabilities due to the radial force associated with elastic stresses along curved streamlines. Recent work has shown that the addition of steady or oscillatory shear flow in a transverse direction (orthogonal superposition) can suppress these instabilities. The present work instead investigates the effect of oscillatory parallel superposition, for the particular case of circular Couette flow. For flow of an Oldroyd-B fluid, the oscillation has a weak stabilizing effect if the oscillation amplitude is not too large. If on the other hand, the oscillation amplitude is such that the angular velocity of the moving cylinder changes sign over part of the cycle, the flow is destabilized. However, in the limit where the motion is purely oscillatory (i.e., large amplitude oscillatory shear), the flow is stabilized relative to steady circular Couette flow with the same maximum shear rate. Finally, in the limit where curvature goes to zero-plane Couette flow-parallel superposition makes the already stable flow slightly more stable, increasing the decay rate of fluctuations in the range of parameters studied. Furthermore, two-dimensional disturbances are the most slowly decaying; we have extended Squire's theorem in this case to encompass an arbitrary time dependence. (C) 2000 American Institute of Physics.
\end{abstract}

[S1070-6631(00)01211-3]

\section{INTRODUCTION}

Beginning with the work of Larson et al., ${ }^{1}$ hydrodynamic instabilities due to elastic effects in inertialess flows have been extensively studied this decade. Their analysis of circular Couette flow of an Oldroyd-B fluid showed a mechanism of instability tied to the development of non-uniform azimuthal normal stresses that resulted in radial motions giving rise to fully three-dimensional flow. The occurrence of this transition was also confirmed experimentally in a Boger fluid. In an Oldroyd-B fluid, the criterion for instability to occur is that $\epsilon^{1 / 2} W e_{\theta}=O(1)$, where $W e_{\theta}$ is the azimuthal Weissenberg number and $\epsilon$ is the gap width, nondimensionalized with respect to the radius of the cylinder. The transition occurs as a Hopf bifurcation with nonaxisymmetric modes $^{2,3}$ being the most dangerous and nonlinear analysis ${ }^{3,4}$ predicting that these modes are subcritical. Experiments by Baumert and Muller ${ }^{5}$ have revealed that these transitions may actually be preceded in $W e_{\theta}$ by a transition to a very weak, axisymmetric cellular flow. A plausible theoretical explanation of this observation has been given by AlMubaiyedh et al. ${ }^{6}$ By including heat generation and nonisothermal viscoelastic effects in their analysis, they predict an

\footnotetext{
a) Author to whom correspondence should be addressed. Electronic mail: graham@engr.wisc.edu
}

instability quite consistent with the experiments. Computations $^{7-9}$ and experiments ${ }^{10}$ show that finite gap effects are stabilizing.

In this work we investigate the effect of superimposing a secondary flow on the circular Couette flow. Recent studies by Graham ${ }^{4}$ and Ramanan et al. ${ }^{11}$ have shown that superposition of flow in the transverse (axial) direction, i.e., orthogonal superposition, has a significant effect on the stability characteristics of the circular Couette flow. In particular, orthogonal superposition may appreciably delay the onset of instability in this flow geometry. A dominant balance analysis with steady axial flow and axial Weissenberg number $W e_{z} \gg 1$ shows that the condition for instability becomes $W e_{\theta}=O\left(\epsilon^{-1 / 2} W e_{z}\right)$ : i.e., the regime of stable flow increases linearly with $W e_{z}$; axial wavelengths $\ell$ of $O(W e)$ are the most unstable. Computationally, we find that this scaling regime begins at roughly $W e_{z}=1$; significant stabilization can be achieved even with an axial shear rate that is smaller than the azimuthal by a factor of $\epsilon^{1 / 2}$. Nonaxisymmetric perturbations, which are actually the most destabilizing in the absence of axial flow, are very strongly suppressed, occurring only when $W e_{\theta}=O\left(\epsilon^{-1 / 2} W e_{z}^{2}\right)$. The scaling analysis also reveals the mechanism of stabilization, which is simple and robust. The axial shear induces axial stresses, particularly an axial tension $\tau_{z z}$. This acts like the tension in a stretched membrane, resisting deformation, with short wavelengths being resisted more than long. The results above reflect how 
large $W e_{\theta}$ and $\ell$ must become for the destabilizing hoop stress term to compete with the axial tension in the leading order momentum balance. In effect, stabilization of the flow is obtained by using viscoelasticity against itself.

In applications, an oscillatory transverse flow is likely to be more feasible than a steady one. In this case an additional parameter, the transverse Deborah number arises, $D e_{z}$ $=\lambda \omega$, where $\omega$ is the angular frequency of the oscillation. Now $W e_{z}$ represents the maximum axial shear rate. We can also arrive at scaling results in this situation. ${ }^{11}$ When $D e_{z}$ $=O(1)$ these collapse to the steady case above. When $D e_{z}$ $=O\left(W e_{\theta}\right)$ and $W e_{z}=O(1)$, corresponding to an $O\left(\epsilon^{1 / 2}\right)$ strain, there is no stabilization. In contrast, when $D e_{z}$ $=O\left(W e_{\theta}\right)$ and $W e_{z}=O\left(\epsilon^{-1 / 2}\right)$, corresponding to an $O(1)$ strain, stabilization is essentially complete: instability does not arise until $W e_{\theta}=O\left(\epsilon^{-1}\right)$ rather than $O\left(\epsilon^{-1 / 2}\right)$. All of these scaling results have been corroborated by numerical linear stability analyses in the circular Couette and Dean geometries. ${ }^{4,11}$ Nevertheless, we also find two regions of instability-when the external forcing frequency is close to the inverse of the relaxation time and when the frequency is close to zero. In both these cases the instability is a synchronous resonance bifurcation, i.e., the frequency of the bifurcating solution equals the forcing frequency. Consistent with the analysis of Davis and Rosenblat ${ }^{12}$ we find that the zero frequency limit is singular with the response of the disturbance containing large temporal increases at time instants close to $1 / 4$ and $3 / 4$ of the time period $T$ of oscillation, corresponding to the instants when the local axial shear rate is close to zero. This singular transient behavior can be understood in a crude sense on the basis of a quasisteady argument. Addition of steady axial flow at small $W e_{z}$ is destabilizing; ${ }^{4}$ accounting for the axial reflection symmetry of the flow, the instantaneous growth rate is thus roughly proportional to $\sin (4 \pi t / T)$. Integrating the growth rate to yield the amplitude gives maxima at $t=T / 4$ and $t=3 T / 4$ as found in the exact computation. We note that in principle this quasisteady approach can be made rigorous in the low frequency limit by application of the WKBJ method.

The present work extends the methodology of Ramanan et al. ${ }^{11}$ to the superposition of parallel flow in the circular Couette geometry by modulation of the inner or outer cylinder rotation rate. This subject has been exhaustively studied for the classical Newtonian Taylor-Couette flow; for a review refer to Donnelly. ${ }^{13}$ In the case when the base flow is generated by the inner cylinder, Hall ${ }^{14}$ showed that modulation of the inner cylinder rotation rate is destabilizing. Given the same base flow, Walsh and Donnelly ${ }^{15}$ showed significant stabilization when the modulation was imposed on the outer cylinder. The stability of the Newtonian fluid in this geometry is governed by the interaction of centrifugal forces generated by base flow and the modulation. For the zero Reynolds number viscoelastic flow that we are analyzing here, we ask whether we can use parallel superposition to stabilize the flow. Further, we seek to understand the interaction between the material time scale given by the relaxation time of the fluid and the external forcing. In particular, it is of interest to investigate whether the parallel superposi-

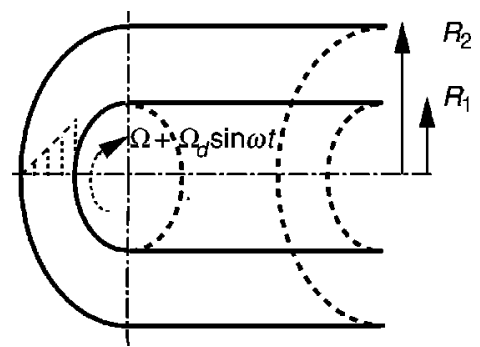

FIG. 1. Schematic diagram of the circular Couette geometry. $\Omega_{d}=0, \Omega$ $\neq 0$ reduces to pure circular Couette flow while $\Omega=0, \Omega_{d} \neq 0$ corresponds to large amplitude oscillatory shear.

tion would result in nontrivial regions of instability that depend on shear rate and frequency, as is the case in orthogonal superposition.

In the limiting case of a narrow gap, and azimuthal wave number of disturbances is on the order of the gap width, the circular Couette flow analysis reduces to that of plane Couette flow. Therefore, some stability results are also presented for parallel superposition in this case. We have also shown that Squire's theorem extends to time-dependent base states in this situation, so two-dimensional disturbances are the most slowly decaying.

\section{FORMULATION}

\section{A. Circular Couette flow}

We consider the inertialess flow of an upper convected Maxwell (UCM) or Oldroyd-B fluid in the annular region between infinitely long circular cylinders. We assume that the annulus is open, i.e., we do not impose a constraint on the net axial flow rate. The fluid has relaxation time $\lambda$; the polymer and solvent contributions to the viscosity are $\eta_{p}$ and $\eta_{s}$, respectively, with the ratio $\eta_{s} / \eta_{p}$ denoted by $S$. The inner cylinder, with radius $R_{1}$, is rotating with angular velocity $\Omega+\Omega_{d} \sin (\omega t)$, while the outer cylinder, with radius $R_{2}$, is stationary (Fig. 1). When $\Omega, \Omega_{d} \neq 0$ we have circular Couette with superimposed parallel Couette (CCPC) flow. Similarly, when $\Omega=0, \Omega_{d} \neq 0$ we recover the large amplitude oscillatory shear (LAOS) limit and with $\Omega_{d}=0, \Omega \neq 0$ we have the pure circular Couette limit.

The dimensionless momentum, constitutive, and continuity equations are

$$
\begin{aligned}
& \boldsymbol{\nabla} \cdot \boldsymbol{\tau}-\boldsymbol{\nabla} p+W e_{\theta} S \boldsymbol{\nabla}^{2} \mathbf{v}=0 \\
& \boldsymbol{\tau}+W e_{\theta}\left(\partial \frac{\boldsymbol{\tau}}{\partial t}+\mathbf{v} \cdot \boldsymbol{\nabla} \boldsymbol{\tau}-\left(\boldsymbol{\tau} \cdot \boldsymbol{\nabla} \mathbf{v}+(\boldsymbol{\tau} \cdot \boldsymbol{\nabla} \mathbf{v})^{\mathbf{T}}\right)\right) \\
& \quad=W e_{\theta}\left(\boldsymbol{\nabla} \mathbf{v}+\boldsymbol{\nabla} \mathbf{v}^{\mathbf{T}}\right) \\
& \boldsymbol{\nabla} \cdot \mathbf{v}=0
\end{aligned}
$$

where $\mathbf{v}$ is the velocity, $p$ is the pressure, and $\boldsymbol{\tau}$ is the polymer stress tensor. No-slip boundary conditions are imposed at the two cylinders. When $\Omega \neq 0$, the azimuthal Weissenberg number, $W e_{\theta}$, is defined by

$$
W e_{\theta}=\frac{\lambda \Omega(1-\epsilon)}{\epsilon},
$$


where

$$
\epsilon=\frac{R_{2}-R_{1}}{R_{2}} .
$$

Similarly, when $\Omega=0$ and $\Omega_{d} \neq 0, W e_{\theta}$ is defined by replacing $\Omega$ with $\Omega_{d}$ in Eq. (4). The Newtonian fluid is recovered for $S \rightarrow \infty$ with the pressure $p$ rescaled accordingly. Note that $\epsilon /(1-\epsilon)$ measures the maximum streamline curvature. Length is scaled with the gap width $R_{2}-R_{1}=\epsilon R_{2}$ and stress with $\eta_{p} / \lambda$. Velocity is scaled with the speed of inner cylinder rotation $(1-\epsilon) R_{2} \Omega$ and time is nondimensionalized by $\epsilon /((1-\epsilon) \Omega)$. The dimensionless flow domain is $\{(r, \theta, z):[(1-\epsilon) / \epsilon]<r<(1 / \epsilon), 0<\theta \leqslant 2 \pi,-\infty$ $<z<\infty\}$. We define a new radial coordinate, $\rho=r+1$ $-1 / \epsilon$, so that $\rho=0$ is the inner cylinder and $\rho=1$ the outer.

Analytical solutions for the base state velocities $\overline{\mathbf{v}}$ and polymer stresses $\overline{\boldsymbol{\tau}}$ for the LAOS and CCPC flow are given in the Appendix. Our interest is in the stability of these solutions to infinitesimal perturbations. We define a vector of perturbations $\quad \mathbf{u}=\left(\tilde{\tau}_{r r}, \tilde{\tau}_{r \theta}, \tilde{\tau}_{r z}, \tilde{\tau}_{\theta \theta}, \tilde{\tau}_{\theta z}, \tilde{\tau}_{z z}, \tilde{v}_{r}, \tilde{v}_{\theta}, \tilde{v}_{z}, \tilde{p}\right)$, where, for example, $\tilde{\tau}_{\theta \theta}=\tau_{\theta \theta}-\bar{\tau}_{\theta \theta}$. It has been shown by Larson et al. ${ }^{1}$ that the relevant regime for instability to occur is when $W e_{\theta}=O\left(\epsilon^{-1 / 2}\right)$. This magnitude of $W e_{\theta}$ is necessary for the destabilizing hoop stress $\tilde{\tau}_{\theta \theta} / r$ to appear in the leading order perturbation momentum balance. We enforce this scaling in our work by defining an $O(1)$ scaled azimuthal Weissenberg number $W p$ such that $W p$ $=\epsilon^{1 / 2} W e_{\theta}$. Following Larson et al. ${ }^{1}$ and Graham, ${ }^{4}$ the perturbations are scaled as follows: $\tilde{\tau}_{r r}=O(1), \tilde{\tau}_{r \theta}$ $=O\left(\epsilon^{-1 / 2}\right), \quad \tilde{\tau}_{r z}=O(1), \tilde{\tau}_{\theta \theta}=O\left(\epsilon^{-1}\right), \tilde{\tau}_{\theta z}=O\left(\epsilon^{-1 / 2}\right), \tilde{\tau}_{z z}$ $=O(1), \quad \tilde{v}_{r}=O\left(\epsilon^{1 / 2}\right), \quad \tilde{v}_{\theta}=O(1), \quad \tilde{v}_{z}=O\left(\epsilon^{1 / 2}\right), \quad$ and $\tilde{p}$ $=O(1)$. This scaling is identical to the leading order scaling of the base state velocities and stresses. Further, as in Ramanan et al., ${ }^{11}$ we rescale to the "low frequency" regime such that $\omega=\omega_{1} \epsilon^{1 / 2}$ and time scale adjusted accordingly to $t=t_{1} \epsilon^{-1 / 2}$, where $\omega_{1}$ and $t_{1}$ are $O(1)$. Setting $\omega_{1}=0$ in this regime gives us the pure circular Couette limit. The "high frequency" regime is obtained for $\omega=O(1)$ or $\omega_{1}$ $=O\left(\epsilon^{-1 / 2}\right)$. Here the forcing frequency is on the order of the azimuthal shear rate.

To analyze the stability of the CCPC flow we expand the perturbation vector using disturbances of the form

$$
\mathbf{u}=\delta \phi(\rho, t) e^{i(\alpha z+n \theta)}+O\left(\delta^{2}\right),
$$

where $\quad \phi=\left(\hat{\tau}_{r r}(\rho, t), \hat{\tau}_{r \theta}(\rho, t), \hat{\tau}_{r z}(\rho, t), \hat{\tau}_{\theta \theta}(\rho, t), \hat{\tau}_{\theta z}(\rho, t)\right.$, $\left.\hat{\tau}_{z z}(\rho, t), \hat{v}_{r}(\rho, t), \hat{v}_{\theta}(\rho, t), \hat{v}_{z}(\rho, t)\right)$ and $\alpha$ and $n$ are axial and azimuthal wave numbers, respectively. It has been shown by Joo and Shaqfeh ${ }^{16}$ for the circular Couette instability that for non-axisymmetric modes to appear in the leading order equations $n$ must be scaled to be $O\left(\epsilon^{-1 / 2}\right)$. We implement this scaling in CCPC flow by defining $\tilde{n}=\epsilon^{1 / 2} n=O(1)$. We also examine the regime where $n=O\left(\epsilon^{-1}\right)$. At these large values of $n, W e_{\theta}$ must be $O\left(\epsilon^{-1}\right)$ for curvature driven instability to occur in the absence of modulation; we include this case since the possibility remains that modulation might drive an instability. To leading order in $\epsilon$, this situation is equivalent to the plane Couette geometry considered next.

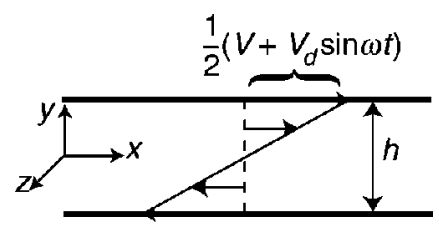

FIG. 2. Schematic diagram of the plane Couette geometry. $V_{d}=0, V \neq 0$ reduces to pure plane Couette flow.

\section{B. Plane Couette flow}

In the plane Couette geometry we consider flow between two infinitely long plates that are moving in the opposite directions (Fig. 2). We find this geometrical arrangement to be computationally more favorable in preserving the symmetry of the solution as compared to one with one plate fixed and the other moving. We use a Cartesian coordinate system with the flow direction denoted by $x$, the gradient direction $y$ and $z$ being the neutral direction. In our modulated system the two plates are moving in the $x$ direction with speeds $\pm \frac{1}{2}\left(V+V_{d} \sin (\omega t)\right) . V \neq 0$ and $V_{d}=0$ gives us the plane Couette flow while nonzero $V$ and $V_{d}$ results in the plane Couette with superimposed parallel Couette (PCPC) flow. As in the circular Couette flow, the ratio of the amplitudes $V_{d} / V$ is defined to be $\chi$. The fluid properties are as defined earlier.

In this geometry, the length is scaled by the gap width between the plates, $h$, and velocity is nondimensionalized with twice the steady plate velocity, $V$. Time is scaled with the shear rate $V / h$ and stress with $\eta_{p} / \lambda$. The conservation equations are given by (1)-(3) in Cartesian coordinates with $W e_{\theta}$ replaced by $W e_{x}$ where

$$
W e_{x}=\frac{\lambda V}{h} .
$$

The dimensionless flow domain is $\{(x, y, z):-\infty<x<\infty$, $-1 / 2<y<1 / 2,-\infty<z<\infty\}$. The base state solutions to flow governed by such kinematics is given in the Appendix. We define the perturbation vector $\mathbf{u}=\left(\tilde{\tau}_{x x}, \tilde{\tau}_{x y}, \tilde{\tau}_{x z}\right.$, $\left.\tilde{\tau}_{y y}, \tilde{\tau}_{y z}, \tilde{\tau}_{z z}, \tilde{v}_{x}, \tilde{v}_{y}, \tilde{v}_{z}, \tilde{p}\right)$. In contrast to the circular geometry, all stresses, velocities, and frequency are specified to be $O(1)$. Linear stability analysis is carried out with disturbances given to be

$$
\mathbf{u}=\delta \phi(y, t) e^{i(k x+m z)}+O\left(\delta^{2}\right) .
$$

As above, $\phi(y, t)$ contains the Fourier mode amplitudes, denoted with carets. By deriving an extension to Squire's theorem to time-dependent viscoelastic base flows, we show that for a given $k$, the least stable mode has $m=0$, allowing us to restrict our numerical analysis to two-dimensional perturbations. We consider $k=O(1)$ here which corresponds to $n$ $=O\left(\epsilon^{-1}\right)$ in the circular geometry.

\section{STABILITY AND NUMERICAL ANALYSIS}

After applying spatial discretization, our problem has the form

$\mathbf{E} \dot{\mathbf{u}}=\mathbf{A}(t) \mathbf{u}$. 
$\mathbf{A}(t)$ is a matrix with time periodic coefficients such that $\mathbf{A}(t)=\mathbf{A}(t+T)$, where $T$ is the period of the forcing function. The stability of this type of problem is determined by the application of Floquet theory. ${ }^{17}$ The solution $\mathbf{u}(T)$ at $t$ $=T$, given the initial vector $\mathbf{u}(0)$, can be written in the form

$$
\mathbf{u}(T)=\boldsymbol{\Phi}(T) \mathbf{u}(0),
$$

so $\boldsymbol{\Phi}(T)$ is the monodromy matrix, whose eigenvalues $\beta$, known as Floquet multipliers, determine the stability of the system. Stable and unstable behavior are indicated by $|\beta|$ $<1$ and $|\beta|>1$, respectively. The Floquet exponent $\sigma$ is defined by the relation

$$
\beta=\exp (\sigma T) .
$$

Suppose $\boldsymbol{\Psi}$ is an eigenvector of $\boldsymbol{\Phi}(T)$ corresponding to the Floquet exponent $\sigma$. Then, it can be shown ${ }^{17}$ that the solution $\mathbf{w}(t)$ to $\mathbf{E} \dot{\mathbf{w}}=\mathbf{A}(t) \mathbf{w}$ with $\mathbf{w}(0)=\boldsymbol{\Psi}$ has a $T$ periodic component $\zeta(t)$ given by

$$
\zeta(t)=e^{-\sigma t} \mathbf{w}(t)
$$

such that

$$
\zeta(t)=\zeta(t+T) .
$$

Thus, the $\zeta(t)$ corresponding to the dominant Floquet multiplier gives us information on the spatial structure and time evolution of the disturbance.

Our goal is to calculate the dominant eigenvalues $\beta$ of the monodromy matrix $\boldsymbol{\Phi}$. We use a primitive variable formulation of the constitutive, momentum, and constitutive equations. Spatial discretization of the equations is carried out via a Chebyshev collocation technique that includes a staggered grid for the pressure to avoid the spurious modes that arise otherwise. Specifically, we use the ChebyshevGauss-Lobatto integration points with $N+1$ modes for the velocities and stresses and the Chebyshev-Gauss points with $N$ modes for pressure. ${ }^{18}$ For CCPC flow we use $N=32$ while the presence of localized boundary layers in the PCPC case necessitates the use of $N=80$. This choice of spatial resolution is found to be adequate. Since our interest is restricted to picking out the dominant eigenvalues of $\boldsymbol{\Phi}(T)$, we take recourse to the Arnoldi method, ${ }^{19}$ to determine the critical eigenvalue. We use the public domain code $\mathrm{ARPACK}^{20}$ to carry out the Arnoldi calculations. The advantage of the Arnoldi method lies in the fact that we do not need to explicitly construct $\boldsymbol{\Phi}(T)$; we need only determine its action on a vector, $\mathbf{q} \leftarrow \boldsymbol{\Phi}(T) \mathbf{p}$. In our problem $\mathbf{q}$ is the solution vector obtained by integrating the time evolution equations for a given initial vector $\mathbf{p}$. The choice of the initial vector $\mathbf{p}$ is arbitrary, with the only requirement being that it satisfies the algebraic components of Eq. (9). In our case, this is ensured by choosing an arbitrary stress profile and solving the linear momentum and continuity equations for the corresponding velocities and pressure. To compute the periodic component $\zeta(t)$, we calculate the disturbance vector $\mathbf{w}(t)$ by integrating the time evolution Eq. (9) with initial conditions given by the eigenvector of the monodromy matrix obtained from ARPACK. This choice of starting value ensures that the periodicity condition given by Eq. (13) is not violated.
The integration of the time-dependent viscoelastic equations is performed using the EVSS decomposition ${ }^{21}$ of the total stress into elastic and viscous components. We use two schemes for the time integration: (a) a fully implicit discretization for UCM calculations and (b) a splitting technique for Oldroyd-B model involving the solution of the Stokes problem followed by an implicit stress update. Using this technique we have been able to obtain stable solutions for $S$ $\gtrsim 0.1$. The time integration and eigenvalue routines were benchmarked against known eigenvalues for the steady case $(\omega=0)$. In addition, the plane Couette flow limit was checked against the exact analytical results of Gorodtsov and Leonov. ${ }^{22}$ Most calculations are performed with a time step $\Delta t=T / 100$, while for some cases, in particular at very low frequencies, we employ increased temporal resolution, $\Delta t$ $=T / 2500$. This high resolution is necessary to ensure the accuracy of the eigenvalue computation, which is checked by monitoring the periodicity of $\zeta(t)$.

\section{RESULTS}

\section{A. Circular Couette flow}

As stated in the introduction, our aim is to determine whether parallel superposition may be used to stabilize the circular Couette instability. Said differently, can we find an amplitude and frequency of the superposition that would result in an increase in the Weissenberg number at which instability is first seen? We begin our study of this question with some a priori scaling arguments and heuristics. From an analysis similar to one presented in Ramanan et al. ${ }^{11}$ we deduce that for $\Omega_{d} / \Omega=O(1)$ and in the high frequency regime $\left(\omega_{1}=O\left(\epsilon^{-1 / 2}\right)\right)$, the method of averaging ${ }^{23}$ reveals that this is essentially equivalent to the circular Couette limit with no superposition-no change in stability occurs. This is validated from our numerical results where we find $|\beta| \rightarrow 1$ with increasing frequency when the parameters chosen yield neutral stability for $\Omega_{d} / \Omega=0$. More generally we note that because of the quadratic dependence of normal stresses on velocity gradient, the time-averaged value of $\tau_{\theta \theta}$ is larger with parallel superposition than without. Because the mechanism for nonaxisymmetric instability involves this quantity, ${ }^{2,24}$ we might expect parallel superposition to be destabilizing. The situation is not quite so simple though, because the mechanism also involves the change in the stress orientation by the base state shear, which now has a zeromean oscillatory component. This complicated situation does not yield to analysis by simple arguments (except at very low frequencies as we describe below), so we proceed to a numerical study.

Our focus here is the effect of parallel superposition at the critical conditions for circular Couette flow with $S=0$ : $W p=3.94, \alpha=5.1$ and $\tilde{n}=0.47$. Notice that we include nonaxisymmetric modes in this work. This is in contrast to our orthogonal superposition studies ${ }^{4,11}$ where scaling arguments and numerical results indicate that nonaxisymmetric modes are strongly suppressed. Since we do not have any such results for the current flow system and given the fact that nonaxisymmetric modes were shown to be most dangerous ${ }^{25}$ we include these in the present analysis. Figure 3 shows the 


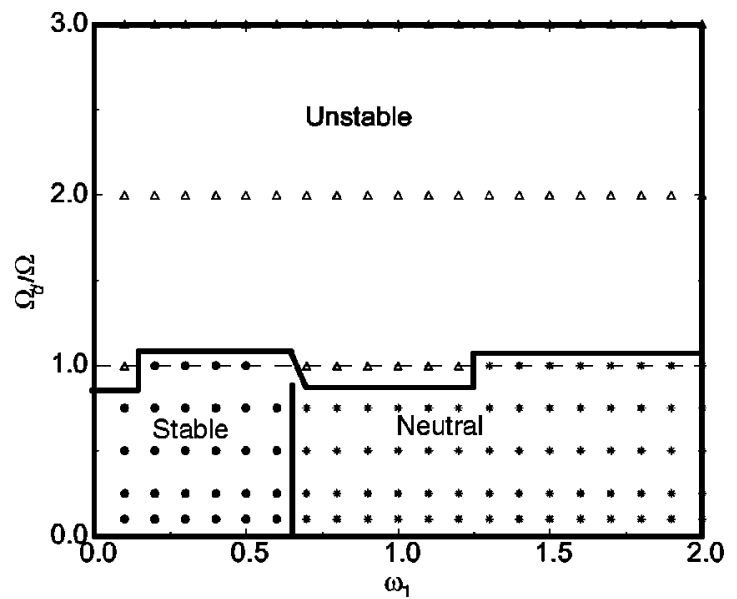

FIG. 3. Stability map for CCPC flow: varying amplitude ratio $\left(\Omega_{d}\right)$ and excitation frequency $\left(\omega_{1}\right)$ for $W p=3.94, \alpha=5.1$, and $\tilde{n}=0.47$ (the point of marginal stability at $\Omega_{d}=0$ ).

stability information for the critical conditions listed above and varying amplitude ratio and excitation frequency. Each marked point represents the result from Floquet analysis. For a chosen value of $\Omega_{d} / \Omega$ and $\omega_{1}$ if the magnitude of the dominant Floquet multiplier is less than one indicating stability we have a - while instability is marked by a $\triangle$. We also show neutrality by a $\star$; here $0.99<|\beta|<1.01$. The plot shows stability for $0<\omega_{1} \lesssim 2 / W p$ and $\Omega_{d} / \Omega<1$. This is qualitatively different from orthogonal superposition where instability was found to occur at $\omega_{1} \approx 1 / W p$ for some superposed shear rates and $\omega_{1} \ll 1 / W p$ for others. Even so, the existence of a distinct stable region is indeed encouraging. If such a scheme is to be of any practical use we have to determine whether we can find an $\Omega_{d} / \Omega$ and $\omega_{1}$ that would result in stabilization of all wavelengths. With $\Omega_{d} / \Omega$ $=0.25$ and $\omega_{1}=0.3$ stability analysis shows $|\beta|<1$ for all axial and azimuthal wave numbers $(2.0 \leqslant \alpha \leqslant 10.0,0.2 \leqslant \tilde{n}$ $\leqslant 1.0$ ) that we have investigated. While we have established that parallel flow superposition is stabilizing, it is of interest to determine the extent of stabilization, i.e., by how much does the critical $W p$ change with the addition of secondary flow. We retain our choice of amplitude and frequency and scan through the parameter space of wave numbers and Weissenberg number to determine the bifurcation point. We find that this occurs at $W p=3.97, \alpha \approx 5.0$ and $\tilde{n} \approx 0.5$. This Weissenberg number is only $0.8 \%$ greater than for the pure circular Couette instability for this amplitude and frequency, significantly lower than the $8 \%$ increase in the critical Weissenberg number seen in the sample case for orthogonal superposition presented in Ramanan et al. ${ }^{11}$ For $\omega_{1}=0.05$, we find $|\beta|=0.8750$ for $W p=3.94, \alpha=5.1$, and $\tilde{n}=0.47$, indicating possibility of increased stabilization. However, we find that at these frequencies, $|\beta|$ increases rapidly with increasing $W p$ resulting in no gain in the extent of stabilization. One more point to be taken from Fig. 3 is that there is no evidence of resonance phenomena-there is no localized bubble in forcing frequency that leads to significant destabilization, in distinct contrast to the orthogonal superposition case.

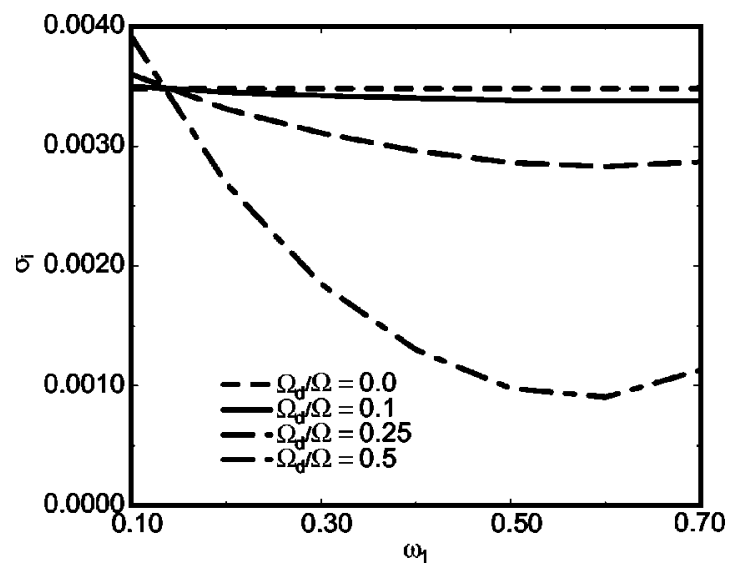

FIG. 4. $\sigma_{i}$ vs $\omega_{1}$ for CCPC flow: $W p=3.94, \alpha=5.1$, and $\tilde{n}=0.47$.

At $\Omega_{d} / \Omega>1, W p=3.94, \alpha=5.1, \tilde{n}=0.47$, and $S=0$ we find instability for all frequencies that we have investigated. At these amplitude ratios the superposition would have the effect of changing the direction of rotation of the inner cylinder for some time instants resulting in a change in the base flow direction-even if stabilization were to occur here, this regime is unlikely to be of practical interest so we have not explored it in detail. The dominant Floquet multiplier at these amplitudes has a nonzero complex part, indicating that the bifurcating solution is quasiperiodic: i.e., this is not a synchronous resonance bifurcation. Recall from Ramanan et al. ${ }^{11}$ that instability for orthogonal superposition always occurs as a synchronous resonance bifurcation with $\sigma_{r}=\sigma_{i}=0$. Figure 4 plots the imaginary part of the complex Floquet exponent $\sigma_{i}$. At lower values of $\Omega_{d} / \Omega$ we find that this is close to the frequency of the bifurcation when there is no forcing. As the amplitude ratio increases, $\sigma_{i}$ decreases while still remaining in the same order of magnitude as $1 / W p$.

Unlike in the orthogonal superposition, we also find relatively large regions of "neutral" stability-the superposition has virtually no effect on the stability characteristics of the system. At low amplitude ratios, the system approaches criticality for all excitation frequencies. This is to be expected

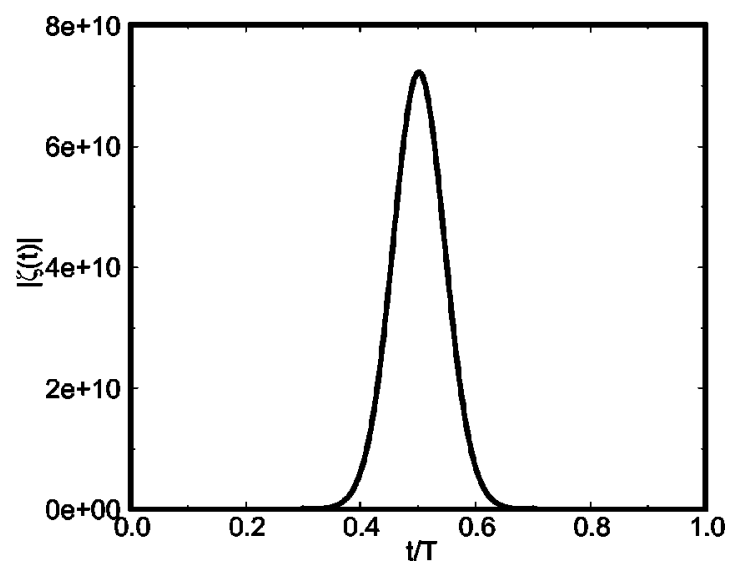

FIG. 5. $|\zeta(t)|$ vs $t / T$ for CCPC flow: $W p=3.94, \alpha=5.1$, and $\tilde{n}=0.47$, $\Omega_{d} / \Omega=0.25, \omega_{1}=0.005$. Note the large increases at $t / T=0.5$. 


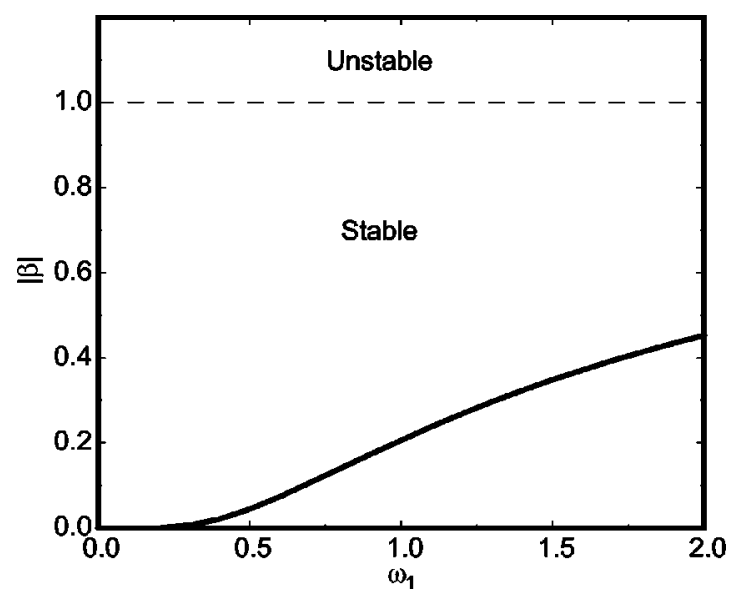

FIG. 6. $\beta$ vs $\omega_{1}$ for LAOS flow: $W p=3.94, \alpha=5.1$, and $\tilde{n}=0.47$. Note that $\beta \ll 1$ for $0<\omega_{1}<0.25$.

since the $\Omega_{d} \rightarrow 0$ limit is regular. However, the same cannot be said for $\omega_{1} \rightarrow 0$. It was shown in Ramanan et al. ${ }^{11}$ that, consistent with the analysis of Davis and Rosenblat, ${ }^{12}$ the zero frequency limit is singular with large increases in the magnitude of the disturbances. Figure 5 shows the large burst occurring at around $t / T=1 / 2$, different from the transverse superposition where we had large increases at $t / T$ $=1 / 4$ and $t / T=3 / 4$. Figure 5 is for $W p=3.94, \Omega_{d} / \Omega$ $=0.25, \alpha=5.1, \tilde{n}=0.47$, and $\omega_{1}=0.005$ which yields $|\beta|$ $=0.2175$. Since $|\beta|<1$, the state is nominally stable, but the huge transient amplification renders the linear stability result moot. As in the orthogonal superposition case, a simple quasisteady argument illuminates the origin of the transient burst: the instantaneous Weissenberg number is larger than the critical value of 3.94 in the first half of the cycle and smaller in the second, so the instantaneous growth rate is roughly proportional to $\sin \omega_{1} t$. Integrating this gives a disturbance amplitude that varies as $-\left(1 / \omega_{1}\right) \cos \omega_{1} t$, which peaks at $T / 2$, as found from the analysis. Unsurprisingly, evidence of this singularity is also seen with instability at $\omega_{1} \rightarrow 0$ for $\Omega_{d} / \Omega>1$.

We also examine the stability characteristics of large amplitude oscillatory shear (LAOS) in a circular geometry. Numerical results indicate that LAOS is significantly stabilized as compared to the base CC flow. In LAOS the steady component of the angular velocity is identically zero which gives a scaled azimuthal shear rate of $W p \sin \left(\omega_{1} t_{1}\right)$. Figure 6 shows $|\beta|$ vs $\omega_{1}$ for $W p=3.94, \alpha=5.1$, and $\tilde{n}=0.47$. Notice that $|\beta| \ll 1$ for $0 \leqslant \omega_{1}<0.3$ indicating a relatively fast decay rate. This is also indicative of the singularity of the zero frequency limit since for $\omega_{1}=0$, a steady analysis yields $|\beta|=1$. Figure 6 shows $|\beta|$ vs $\omega_{1}$ for $0<\omega_{1} \leqslant 2$. We find a monotonic increase in $\beta$ that is still well below criticality. In fact, we find that for $\omega_{1}>10,|\beta| \rightarrow 1$. Clearly, instability relative to the CC limit is not an issue in this flow. Further, computations show that for $\omega_{1}=0.2538(=1 / W p),|\beta| \ll 1$ for $3.1 \leqslant \alpha \leqslant 7.1$ and $0.37 \leqslant \tilde{n} \leqslant 0.57$. Here $|\beta|$ varies from a low of $2.659 \times 10^{-3}$ to a high of $4.407 \times 10^{-3}$. It appears that the flow is strongly stabilized for all disturbance wavelengths. Interestingly, investigation of the Floquet exponents

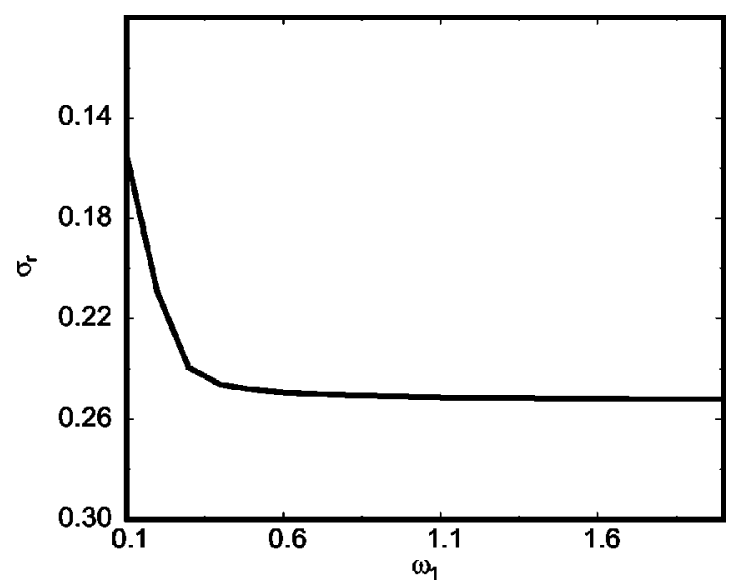

FIG. 7. $\sigma_{r}$ vs $\omega_{1}$ for LAOS flow: $W p=3.94, \alpha=5.1$, and $\tilde{n}=0.47 . \sigma_{r} \approx$ -0.251 for $\omega_{1}>0.5$.

reveals that the decay rate $\sigma_{r} \approx-0.251$ for $\omega_{1}>0.5$ (Fig. 7) while $\sigma_{i} \approx 0$ for all $\omega_{1}$.

\section{B. Plane Couette flow}

Tlapa and Bernstein ${ }^{26}$ developed the viscoelastic analogue of Squire's theorem for steady plane shear flow of an Oldroyd-B fluid. It is straightforward to generalize this result to a time-dependent inertialess plane shear flow. If the following variable changes are made in the linearized equations governing the perturbations:

$$
\begin{aligned}
& K^{2}:=k^{2}+m^{2}, \\
& \hat{T}_{x x}:=\frac{1}{K^{2}}\left(k^{2} \hat{\tau}_{x x}+2 k m \hat{\tau}_{x z}+m^{2} \hat{\tau}_{z z}\right), \\
& \hat{T}_{y x}:=\frac{1}{K}\left(k \hat{\tau}_{y x}+m \hat{\tau}_{y z}\right), \\
& \hat{T}_{y y}:=\hat{\tau}_{y y}, \\
& \hat{U}:=\frac{1}{K}\left(k \hat{v}_{x}+m \hat{v}_{z}\right), \\
& \hat{V}:=\hat{v},
\end{aligned}
$$

then the stability problem is seen to be equivalent to a twodimensional problem with a base velocity and polymer shear stress reduced by a factor of $k / K$ and normal stress reduced by $(k / K)^{2}$. These reductions in the base state quantities are equivalent to a rescaling of the Weissenberg number by $k / K$ as can easily be seen from the equations governing the timedependent base state. Since the three-dimensional problem is equivalent to a two-dimensional problem at a lower Weissenberg number, we only present results for the twodimensional case.

We re-emphasize (from Sec. III) that the presence of boundary layers in the stability problem for plane Couette flow necessitates the use of large number of modes to accurately determine the dominant eigenvalue. This problem is compounded by the slow convergence of the continuous portion of the spectrum, due to the nonintegrable singularities in 


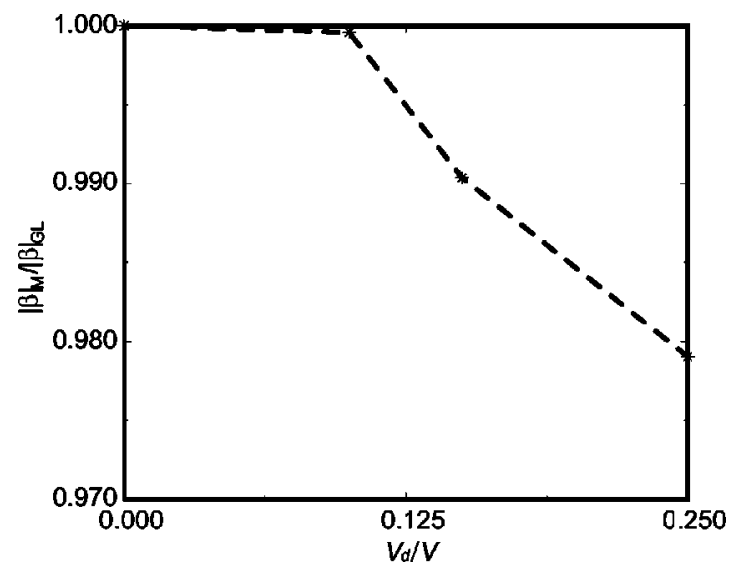

FIG. 8. $|\beta|_{M} /|\beta|_{\mathrm{GL}}$ for PCPC flow: $W e_{x}=5.0, k=1.0, \omega_{1}=0.2$.

the stress components of these models. ${ }^{4}$ A large number of Chebyshev modes is thus required to distinguish the continuous and discrete portions of the spectrum. This problem grows more severe as $W e_{x}$ and $k$ increase, so we limit our investigation to wave number $k=1$. The accurate resolution of the spectrum is also dependent on the time step used in our time-integration procedure. We have found that for $W e_{x}=5$ and $k=2, \Delta t=T / 100$ with $N=120$ does not resolve the eigenvalues satisfactorily. Notwithstanding this limitation, we are still able to gain qualitative information about the stability of the flow. This is done by tracking the evolution of the discrete Gorodtsov-Leonov ${ }^{4,22}$ (GL) modes that occur in the steady plane Couette flow as secondary flow is added. While the GL modes are stable in plane shear flows, the addition of secondary flow has been known to lead to instability. This was shown in Graham ${ }^{4}$ for circular Couette flow with superposed axial Couette flow where the instability was traced to one of GL modes, which became more unstable with increasing azimuthal shear rate for a fixed axial flow rate. Similarly, does parallel flow oscillation stabilize or destabilize the GL mode? The simulations presented here are for $W e_{x}=5, S=0.1, \omega=0.2$, and $k=1$ and varying amplitude ratio $V_{d} / V$. The nonzero solvent viscosity is used here because it allows the use of a splitting method for timeintegration, providing significant computational savings for the high-resolution simulations required. Figure 8 shows the ratio of $|\beta|$ corresponding to the discrete mode in the forced system $\left(|\beta|_{M}\right)$ to that for the steady GL mode $|\beta|\left(|\beta|_{G L}\right)$ for $k=1$ and varying $V_{d} / V$. We find that increasing the amplitude ratio results in a slight, albeit increased stabilization of the flow.

\section{CONCLUSIONS}

We have shown that parallel flow superposition can have a slight stabilizing effect on viscoelastic circular Couette flow instability, if the frequency and amplitude of oscillation are small and may also slightly increase the decay rate of perturbations in plane Couette flow. The extent of stabilization achieved is significantly smaller than with orthogonal superposition and is limited to a smaller region of parameter space. Therefore parallel superposition cannot be viewed as a robust method for suppression of elastic instability. A basic reason for the failure of parallel superposition relative to orthogonal is simply this: orthogonal superposition introduces axial stresses into the radial perturbation momentum balance, providing the possibility for the dominant balance there to change. As described in the introduction, this is ultimately what occurs, as the destabilizing hoop stress term drops out of the dominant balance as axial Weissenberg number increases. Parallel superposition introduces no such change and correspondingly has very little effect until the oscillatory part of the flow becomes larger than the steady.

\section{ACKNOWLEDGMENTS}

This work was supported by an NSF CAREER Award No. CTS-9502677 and a 3M Non-Tenured Faculty Award.

\section{APPENDIX: BASE STATE SOLUTIONS}

CCPC: The equations correspond to the regime where $\omega=O\left(\epsilon^{1 / 2}\right)$ and $t=O\left(\epsilon^{-1 / 2}\right)$ with all other variables scaled as in Sec. II. We define $\omega_{1}=\epsilon^{-1 / 2} \omega=O(1)$ and $t_{1}=\epsilon^{1 / 2} t=O(1)$,

$$
\begin{aligned}
& \bar{v}_{r}=0, \\
& \bar{v}_{\theta}=(1-\rho)\left(1+\chi \sin \left(\omega_{1} t_{1}\right)\right), \\
& \bar{v}_{z}=0, \\
& \bar{\tau}_{r r}=0, \\
& \bar{\tau}_{r \theta}=-\frac{W p\left(1+\omega_{1}^{2} W p^{2}-\omega_{1} \chi W p \cos \left(\omega_{1} t_{1}\right)+\chi \sin \left(\omega_{1} t_{1}\right)\right)}{\left(1+\omega_{1}^{2} W p^{2}\right)}, \\
& \bar{\tau}_{r z}=0,
\end{aligned}
$$




$$
\begin{aligned}
\bar{\tau}_{\theta \theta}= & W p^{2}\left[\frac{2+\chi^{2}+12 \omega_{1}^{2} W p^{2}+5 \omega_{1}^{2} \chi^{2} W p^{2}+18 \omega_{1}^{4} W p^{4}+4 \omega_{1}^{4} \chi^{2} W p^{4}+8 \omega_{1}^{6} W p^{6}}{\left(1+\omega_{1}^{2} W p^{2}\right)\left(1+4 \omega_{1}^{2} W p^{2}\right)}\right. \\
& -\frac{6 \omega_{1} \chi W p \cos \left(\omega_{1} t_{1}\right)-26 \omega_{1}^{3} \chi W p^{3} \cos \left(\omega_{1} t_{1}\right)-8 \omega_{1}^{5} \chi W p^{5} \cos \left(\omega_{1} t_{1}\right)}{\left(1+\omega_{1}^{2} W p^{2}\right)\left(1+4 \omega_{1}^{2} W p^{2}\right)} \\
& -\frac{\chi^{2} \cos \left(2 \omega_{1} t_{1}\right)+\omega_{1}^{2} \chi^{2} W p^{2} \cos \left(2 \omega_{1} t_{1}\right)+2 \omega_{1}^{4} \chi^{2} W p^{4} \cos \left(2 \omega_{1} t_{1}\right)+4 \chi \sin \left(\omega_{1} t_{1}\right)}{\left(1+\omega_{1}^{2} W p^{2}\right)\left(1+4 \omega_{1}^{2} W p^{2}\right)} \\
& \left.+\frac{16 \omega_{1}^{2} \chi W p^{2} \sin \left(\omega_{1} t_{1}\right)-3 \omega_{1} \chi^{2} W p \sin \left(2 \omega_{1} t_{1}\right)-3 \omega 1^{3} \chi^{2} W p^{3} \sin \left(2 \omega_{1} t_{1}\right)}{\left(1+\omega_{1}^{2} W p^{2}\right)\left(1+4 \omega_{1}^{2} W p^{2}\right)}\right], \\
\bar{\tau}_{\theta z}= & 0, \\
\bar{\tau}_{z z}= & 0 .
\end{aligned}
$$

LAOS: The frequency and wave number scalings are the same as in the CCPC case,

$$
\begin{aligned}
& \bar{v}_{r}=0, \\
& \bar{v}_{\theta}=(1-\rho) \sin \left(\omega_{1} t_{1}\right), \\
& \bar{v}_{z}=0, \\
& \bar{\tau}_{r r}=0, \\
& \bar{\tau}_{r \theta}=-\frac{W p\left(\cos \left(\omega_{1} t_{1}\right)+\omega_{1} W p \sin \left(\omega_{1} t_{1}\right)\right)}{\left(1+\omega_{1}^{2} W p^{2}\right)}, \\
& \bar{\tau}_{r z}=0, \\
& \bar{\tau}_{\theta \theta}=\frac{W p^{2}\left(1+4 \omega_{1} W p^{2}+\cos \left(2 \omega_{1} t_{1}\right)+2 \omega_{1}^{2} W p^{2} \cos \left(2 \omega_{1} t_{1}\right)-3 \omega_{1} W p \sin \left(2 \omega_{1} t_{1}\right)\right)}{\left(1+\omega_{1}^{2} W p^{2}\right)\left(1+4 \omega_{1}^{2} W p^{2}\right)}, \\
& \bar{\tau}_{\theta z}=0, \\
& \bar{\tau}_{z z}=0 .
\end{aligned}
$$

PCPC: We have $\omega=\omega_{1}=O(1)$ and $W e_{x}=0(1)$,

$$
\begin{aligned}
\bar{v}_{x}= & (1-y)\left(1+\chi \sin \left(\omega_{1} t_{1}\right)\right), \\
\bar{v}_{y}= & 0, \\
\bar{v}_{z}= & 0, \\
\bar{\tau}_{x x}= & W e_{x}^{2}\left[\frac{2+\chi^{2}+12 \omega_{1}^{2} W e_{x}^{2}+5 \omega_{1}^{2} \chi^{2} W e_{x}^{2}+18 \omega_{1}^{4} W e_{x}^{4}+4 \omega_{1}^{4} \chi^{2} W e_{x}^{4}+8 \omega_{1}^{6} W e_{x}^{6}}{\left(1+\omega_{1}^{2} W e_{x}^{2}\right)\left(1+4 \omega_{1}^{2} W e_{x}^{2}\right)}\right. \\
& -\frac{6 \omega_{1} \chi W e_{x} \cos \left(\omega_{1} t_{1}\right)-26 \omega_{1}^{3} \chi W e_{x}^{3} \cos \left(\omega_{1} t_{1}\right)-8 \omega_{1}^{5} \chi W e_{x}^{5} \cos \left(\omega_{1} t_{1}\right)}{\left(1+\omega_{1}^{2} W e_{x}^{2}\right)\left(1+4 \omega_{1}^{2} W e_{x}^{2}\right)} \\
& -\frac{\chi^{2} \cos \left(2 \omega_{1} t_{1}\right)+\omega_{1}^{2} \chi^{2} W e_{x}^{2} \cos \left(2 \omega_{1} t_{1}\right)+2 \omega_{1}^{4} \chi^{2} W e_{x}^{4} \cos \left(2 \omega_{1} t_{1}\right)+4 \chi \sin \left(\omega_{1} t_{1}\right)}{\left(1+\omega_{1}^{2} W e_{x}^{2}\right)\left(1+4 \omega_{1}^{2} W e_{x}^{2}\right)} \\
& \left.+\frac{16 \omega_{1}^{2} \chi W e_{x}^{2} \sin \left(\omega_{1} t_{1}\right)-3 \omega_{1} \chi^{2} W e_{x} \sin \left(2 \omega_{1} t_{1}\right)-3 \omega 1^{3} \chi^{2} W e_{x}^{3} \sin \left(2 \omega_{1} t_{1}\right)}{\left(1+\omega_{1}^{2} W e_{x}^{2}\right)\left(1+4 \omega_{1}^{2} W e_{x}^{2}\right)}\right],
\end{aligned}
$$




$$
\begin{aligned}
& \bar{\tau}_{x y}=-\frac{W e_{x}\left(1+\omega_{1}^{2} W e_{x}^{2}-\omega_{1} \chi W e_{x} \cos \left(\omega_{1} t_{1}\right)+\chi \sin \left(\omega_{1} t_{1}\right)\right)}{\left(1+\omega_{1}^{2} W e_{x}^{2}\right)}, \\
& \bar{\tau}_{x z}=0, \\
& \bar{\tau}_{y y}=0, \\
& \bar{\tau}_{y z}=0, \\
& \bar{\tau}_{z z}=0 .
\end{aligned}
$$

${ }^{1}$ R. G. Larson, E. S. G. Shaqfeh, and S. J. Muller, “A purely elastic instability in Taylor-Couette flow,'” J. Fluid Mech. 218, 573 (1990).

${ }^{2}$ Y. L. Joo and E. S. G. Shaqfeh, "Observations of purely elastic instabilities in the Taylor-Dean flow of a Boger fluid,' J. Fluid Mech. 262, 27 (1994).

${ }^{3}$ R. Sureshkumar, A. N. Beris, and M. Avgousti, "Non-axisymmetric subcritical bifurcations in viscoelastic Taylor-Couette flow," Proc. R. Soc. London, Ser. A 447, 135 (1994).

${ }^{4}$ M. D. Graham, "Effect of axial flow on viscoelastic Taylor-Couette instability," J. Fluid Mech. 360, 341 (1998).

${ }^{5}$ B. M. Baumert and S. J. Muller, "Flow visualization of the elastic Taylor-Couette instability in Boger fluids," Rheol. Acta 34, 147 (1995). ${ }^{6}$ U. A. Al-Mubaiyedh, R. Sureshkumar, and B. Khomami, "Influence of energetics on the stability of viscoelastic Taylor-Couette flow," Phys. Fluids 11, 3209 (1999).

${ }^{7}$ P. J. Northey, R. C. Armstrong, and R. A. Brown, "Finite-amplitude timeperiodic states in viscoelastic Taylor-Couette flow described by the UCM model," J. Non-Newtonian Fluid Mech. 42, 117 (1992).

${ }^{8}$ M. Avgousti, B. Liu, and A. N. Beris, "Spectral methods for the viscoelastic time-dependent flow equations with applications to TaylorCouette flow," Int. J. Numer. Methods Fluids 17, 49 (1993).

${ }^{9} \mathrm{M}$. Avgousti and A. N. Beris, "Non-axisymmetric modes in the viscoelastic Taylor-Couette flow," J. Non-Newtonian Fluid Mech. 50, 225 (1993).

${ }^{10}$ E. S. G. Shaqfeh, S. J. Muller, and R. G. Larson, "The effects of gap width and dilute solution properties on the viscoelastic Taylor-Couette instability,"' J. Fluid Mech. 235, 285 (1992).

${ }^{11}$ V. V. Ramanan, K. A. Kumar, and M. D. Graham, "Stability of viscoelastic shear flows subjected to steady or oscillatory transverse flow,' J. Fluid Mech. 379, 255 (1999).

${ }^{12} \mathrm{~S}$. H. Davis and S. Rosenblat, "On bifurcating periodic solutions at low frequency,"' Stud. Appl. Math. 57, 59 (1977).

${ }^{13}$ R. J. Donnelly, "Externally modulated hydrodynamic system,"' in Non- linear Evolution of Spatio-Temporal Structures in Dissipative Continuous Systems, edited by F. H. Busse and L. Kramer (Plenum, New York, 1990).

${ }^{14} \mathrm{P}$. Hall, "The stability of unsteady cylinder flows," J. Fluid Mech. 67, 29 (1975).

${ }^{15}$ T. J. Walsh and R. J. Donnelly, “Taylor-Couette flow with periodically corotated and counterrotated cylinders," Phys. Rev. Lett. 60, 700 (1988).

${ }^{16}$ Y. L. Joo and E. S. G. Shaqfeh, "A purely elastic instability in Dean and Taylor-Dean flow," Phys. Fluids A 4, 524 (1992).

${ }^{17}$ G. Iooss and D. D. Joseph, Elementary Stability and Bifurcation Theory (Springer, New York, 1990).

${ }^{18}$ C. Canuto, M. Y. Hussaini, A. Quarteroni, and T. A. Zang, Spectral Methods in Fluid Dynamics (Springer, New York, 1988).

${ }^{19} \mathrm{~W}$. E. Arnoldi, "The principle of minimized iteration in the solution of matrix eigenvalue problems,", Q. Appl. Math. 9, 17 (1951).

${ }^{20}$ R. B. Lehoucq, D. C. Sorensen, and C. Yang, ARPACK Users Guide: Solution of Large Scale Eigenvalue Problems by Implicitly Restarted Arnoldi Methods, 1997, ftp://ftp.caam.rice.edu/pub/software/ARPACK.

${ }^{21}$ D. Rajagopalan, R. C. Armstrong, and R. A. Brown, "Finite element methods for calculation of steady, viscoelastic flow using constitutive equations with a Newtonian viscosity,' J. Non-Newtonian Fluid Mech. 36, 159 (1990).

${ }^{22}$ V. A. Gorodtsov and A. I. Leonov, "On a linear instability of a plane parallel Couette flow of viscoelastic fluid,"' J. Appl. Math. Mech. 31, 310 (1967).

${ }^{23}$ J. A. Sanders and F. Verhulst, Averaging Methods in Nonlinear Dynamical Systems (Springer, New York, 1985).

${ }^{24}$ E. S. G. Shaqfeh, "Purely elastic instabilities in viscometric flows," Annu. Rev. Fluid Mech. 28, 129 (1996).

${ }^{25}$ Y. L. Joo and E. S. G. Shaqfeh, "Viscoelastic Poiseuille flow through a curved channel: A new elastic instability," Phys. Fluids A 3, 1691 (1991).

${ }^{26} \mathrm{G}$. Tlapa and B. Bernstein, "Stability of a relaxation-type viscoelastic fluid with slight elasticity," Phys. Fluids 13, 565 (1970). 